\title{
Clostridium difficile Infection Hospitalizations in the United States: Insights From the 2017 National Inpatient Sample
}

\author{
Dhanshree Solanki ${ }^{\mathrm{a}}$, Asim Kichloo ${ }^{\mathrm{b}}$, Zain El-Amir ${ }^{\mathrm{b}}$, Dushyant Singh Dahiya ${ }^{\mathrm{b}}$, \\ Jagmeet Singh ${ }^{c}$, Farah Wani ${ }^{\text {, }}$, Shantanu Solanki ${ }^{c}$, e
}

\begin{abstract}
Background: There is a paucity of contemporary national estimates for Clostridium difficile infection (CDI) hospitalizations by age group, sex, and region in the US population. Also, there is lack of contemporary national statistics on CDI hospitalizations with comorbidities.
\end{abstract}

Methods: We analyzed the 2017 National Inpatient Sample (NIS) to determine the population-based rates of CDI hospitalizations, characteristics of hospitalizations with CDI, and the rates of comorbidities associated with CDI hospitalizations.

Results: There were 329,460 CDI-related hospitalizations in 2017 (almost $1 \%$ of all hospitalizations). The average age for patients admitted with CDI as a principal or secondary diagnosis was 64.7 years (almost 20 years older when compared with all other hospitalizations). Patients 85 years and older had the highest rate of CDI hospitalizations ( 716 per 100,000 hospitalizations), and patients less than 18 years of age had the lowest rate ( 12 per 100,000 hospitalizations). There was a progressive increase in the CDI hospitalization rates with each successive age group. The hospitalization rates were higher in females (114 per 100,000 hospitalizations) than males (88 per 100,000 hospitalizations). The CDI hospitalization rate was highest in the Northeast (109 per 100,000 hospitalizations) and lowest in the West ( 84 per 100,000 hospitalizations). Fluid and electrolyte disturbance $(63.3 \%)$ and renal failure (33.4\%) were the two most common comorbidities associated with CDI hospitalizations. When CDI is a secondary diagnosis, major loss of function, extreme likelihood of dying, septicemia, and septic shock were more common in comparison to CDI as a principal diagnosis.

Conclusions: CDI hospitalization rates were highest in the elderly

Manuscript submitted February 13, 2021, accepted March 25, 2021

Published online April 21, 2021

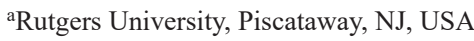

bDepartment of Medicine, Central Michigan University, Saginaw, MI, USA 'Department of Medicine, Geisinger Commonwealth School of Medicine, Scranton, PA, USA

${ }^{\mathrm{d} D e p a r t m e n t ~ o f ~ M e d i c i n e, ~ S a m a r i t a n ~ M e d i c a l ~ C e n t e r, ~ W a t e r t o w n, ~ N Y, ~ U S A ~}$

${ }^{e}$ Corresponding Author: Shantanu Solanki, Department of Medicine, Geisinger Commonwealth School of Medicine, Scranton, PA, USA.

Email: Shan4788@gmail.com

doi: https://doi.org/10.14740/gr1371 over 85 years old and declined with successive decreases in age. Women had higher CDI hospitalization rates than men, and fluid and electrolyte disturbances and renal failure were the most common comorbid conditions. The presence of CDI as a comorbid condition at the time of hospitalization for other principal diagnoses or development of CDI during a hospitalization for other principal diagnoses significantly increases the risk of in-hospital morbidity and mortality.

Keywords: Clostridium difficile; Hospitalizations; Mortality

\section{Introduction}

Clostridium difficile (C. difficile) is a gram-positive anaerobic spore-forming bacillus that infects and colonizes the colon and causes a variety of illnesses, including diarrhea and pseudomembranous colitis, with ranging severities [1]. It is the leading cause of healthcare-related diarrhea, with $70-80 \%$ of C. difficile infections (CDIs) associated with healthcare facility exposure; and it is very well known that infection with $C$. difficile is related to antimicrobial use [2,3]. CDI rates doubled from 2000 to 2010; and in 2011, the incidence of CDI in the USA was reported to be 147.2 cases per 100,000 persons $[4,5]$. Rises in incidence, severity, and mortality were attributed to the epidemic strain ribotype 027 (which was formerly referred to as NAP1/BI/027), which arose in the early $2000 \mathrm{~s}$ and has resulted in outbreaks in Asia, Europe, Canada, and the USA [4]. The strain is highly resistant to fluoroquinolones, produces a toxin that was not common in previous $C$. difficile strains, and produces more toxins A and B than other strains of C. difficile [4]. CDI is a major cause of death in the USA [5, 6]. Between 1999 and 2012 alone, deaths due to C. difficile grew by an almost 10-fold amount, growing from 793 to 7,739 [5].

CDIs occur secondary to alterations in intestinal microbiomes [4]. The bacteria are transmitted through the fecal-oral route, but acquisition can occur from person-to-person contact, contact with the hands of transiently colonized healthcare persons, or exposure to contaminated equipment or surfaces [4]. C. difficile produces toxin A, which causes increased fluid secretion and intestinal permeability, and toxin $\mathrm{B}$, which causes intense colonic inflammation [7]. Infection risk is much higher in hospitalized persons than non-hospitalized persons, but $C$. difficile is estimated to cause 51.9 episodes of communityassociated infection per 100,000 people [4]. Once the bacteria 
colonize the host, certain factors are known to favor symptomatic disease development, the most common of which is the use of multiple types of antibiotics [4]. Antibiotics are related to CDI because antibiotic treatment disrupts the normal flora of the intestinal tract, which can lead to infection [8]. Exposure to certain antibiotics has been related to strain emergence, including the clindamycin-resistant "J strain," which emerged largely due to clindamycin use, and the ribotype 027, which emerged largely due to fluoroquinolone use [4]. Other antibiotics that have been linked to CDI include carbapenems and third- and fourth-generation cephalosporins, but almost all antibiotics carry some risk for gut microbiome disruption [4].

CDI has grown in the USA, and previous literature has characterized CDI through the years [9]. Previous literature reported a rise in deaths from 5.7 to 23.7 deaths per million (from 1999 to 2004) in the USA, with increasing mortality suggested to be attributable to the rise in highly virulent strains of $C$. difficile [10]. Rates were also shown to be higher for whites than other racial groups from 1999 to 2004 [10]. One study examining data from the National Inpatient Sample (NIS) from 2000 to 2005 showed a 23\% annual increase in $C$. difficile-associated hospitalizations and that hospitalizations more than doubled in all age groups except for the youngest age group, which had a $74.1 \%$ increase from 2000 to 2005 [9]. While there are data characterizing hospitalizations for CDI in the recent past, our study contributes contemporary estimates from the NIS database in order to guide clinical management.

The healthcare burden of CDI is undeniable, with CDI estimating to cost $\$ 4.8$ billion in acute care facilities alone and several billion dollars annually $[2,6]$. Previous studies have reported on and characterized the burden of CDI, but there is a lack of contemporary national estimates for CDI hospitalizations and their outcomes. The aim of this study is to examine rates of hospitalizations, characteristics of hospitalizations, and rates of comorbid conditions for CDI hospitalizations using data from the NIS.

\section{Materials and Methods}

\section{Data source and statistical analysis}

The NIS is the largest publicly available inpatient database developed for the Healthcare Cost and Utilization Project (HCUP). It is designed to produce US regional and national estimates of inpatient diseases, resource utilization, and outcomes $[11,12]$. This limited dataset has safeguards in place to protect the privacy of patient data. Institutional Review Board (IRB) approval is not required under the Health Insurance Portability and Accountability (HIPAA) when this dataset is used for analysis [13]. Our study was thus exempt from an IRB review. The estimates for this study were derived from the HCUP 2017 NIS. The temporal trends were drawn from 2010 to 2017 NIS. The data on estimates by age, gender, and region were obtained from the US Census Bureau's Population Estimates Program (PEP) [14]. These web links for these files can be found here (Supplementary Material 1, www.gastrores. org). In the NIS database, the first listed diagnosis is the prin- cipal diagnosis defined as the condition chiefly responsible for resulting in the admission of the patient to the hospital for care [15]. Secondary diagnoses are coexisting clinical conditions at the time of hospital admission or those that develop during the patient's hospital stay. Since the fourth quarter of 2015, HCUP records report diagnoses based on the International Classification of Diseases, Tenth Revision, Clinical Modification (ICD-10-CM). It is a uniform and standardized coding system, which defines diseases, injuries, and other related health conditions in a hierarchical and comprehensive fashion using alphanumeric codes [16]. The Clinical Classifications Software (CCS) provides a system for clustering clinical diagnoses and procedures into meaningful categories [17]. The ICD-10-CM codes and CCS codes used for data analysis can be found here (Supplementary Material 2, www.gastrores.org). The description of variables, such as region, insurance status, severity of illness, and risk of mortality are depicted here (Supplementary Material 1, www.gastrores.org). We used SAS 9.3 (SAS Institute, Cary, NC, USA) software for the analysis. We compared categorical variables by using Chi-squared $\left(\chi^{2}\right)$ test and continuous variables by Wilcoxon rank-sum test. These statistical methods have been used previously in multiple NIS based studies [18-20]. All the differences between the estimates are statistically significant $(\mathrm{P}<0.05)$ unless otherwise specified.

\section{Results}

\section{Baseline characteristics of CDI hospitalizations}

There were 329,460 CDI-related hospitalizations in 2017. This accounted for $0.9 \%$ of all hospitalizations in 2017 . Out of these hospitalizations, $29.3 \%$ had CDI as a principal diagnosis and $70.7 \%$ of the hospitalizations had CDI as a secondary diagnosis. The number of all CDI-related hospitalizations increased from 324,000 in 2010 to 329,500 in 2017 accounting for a $1.7 \%$ increase (Fig. 1). However, the rate of all CDI hospitalizations per 100,000 population declined by $3.3 \%$ over this period (Fig. $2)$. Interestingly, a steady decline was noted in the number as well as rate of all CDI hospitalizations starting 2015. The average age for patients admitted with CDI as a principal or secondary diagnosis was 64.7 years. For all CDI hospitalizations, patients were more likely to be females $(57.1 \%)$. The percent gap between females and males widened when CDI hospitalizations were considered as a primary diagnosis $(63.7 \%$ females vs. $36.3 \%$ males). Medicare covered the highest percent of all CDI hospitalizations (65.6\%), followed by private insurance (19\%) and Medicaid (13.3\%). Around 2\% of the hospitalizations were not covered by insurance. The mean length of stay (LOS) for CDI hospitalizations as a secondary diagnosis was more than double the LOS for hospitalizations with CDI as a principal diagnosis (11.8 days vs. 5.4 days).

\section{Rates of CDI hospitalizations per 100,000 population}

The CDI hospitalization rates per 100,000 population by age, sex, and region are depicted in Figure 3. We intention- 


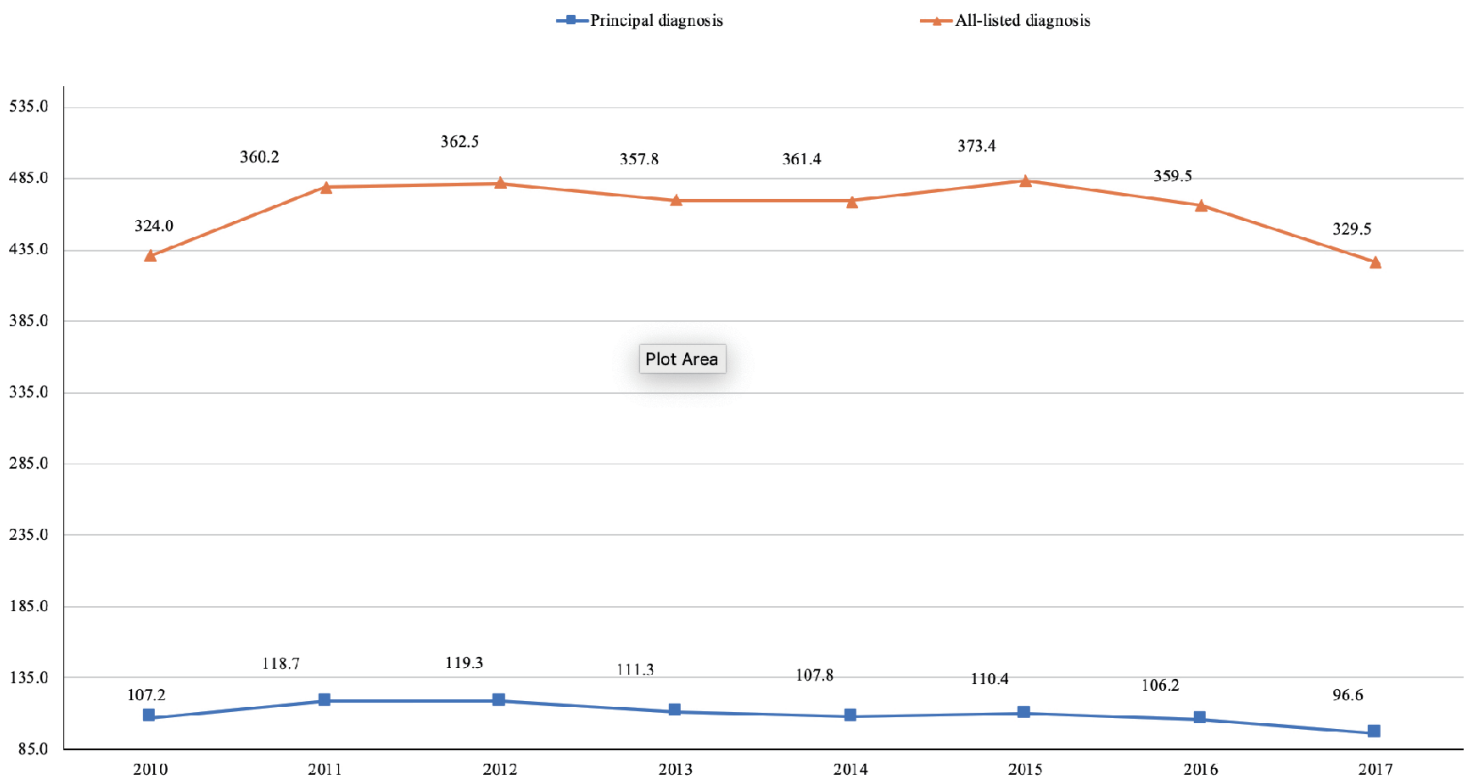

Figure 1. CDI hospitalization trends, 2010 - 2017. The number of hospitalizations with CDI as a principal or secondary diagnosis is depicted in the figure. CDI: Clostridium difficile infection.

ally did not calculate hospitalization rates by race because of missing data in the NIS dataset. The data on race are missing in about 4\% of hospital discharges in the 2017 NIS. The percentage of missing race values was higher in NIS database from previous years. Thus, race-specific estimates from NIS may be biased [21]. Patients 85 years and older had the highest rate of CDI hospitalizations (716 per 100,000 hospitaliza- tions), and patients less than 18 years of age had the lowest rate (12 per 100,000 hospitalizations). There was a rise in the CDI hospitalization rates with each successive age group. The hospitalization rates were higher in females (114 per 100,000 hospitalizations) than males ( 88 per 100,000 hospitalizations). The CDI hospitalization rate was highest in the Northeast (109 per 100,000 hospitalizations) and lowest in the West (84 per

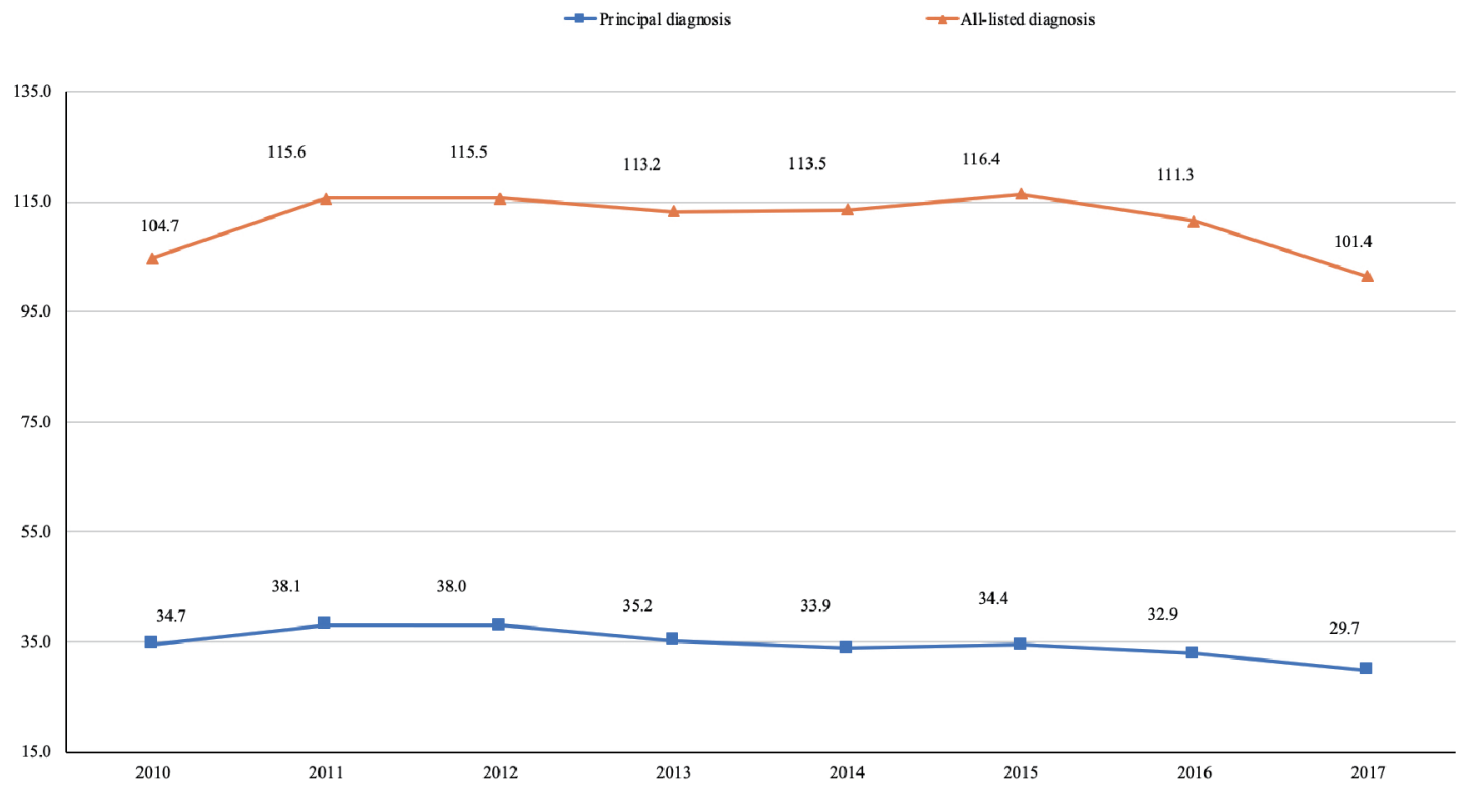

Figure 2. Trends in CDI hospitalizations per 100,000 population, $2010-2017$. The yearly rates of hospitalizations with CDI as a principal or secondary diagnosis per 100,000 US population are depicted in the figure. CDI: Clostridium difficile infection. 


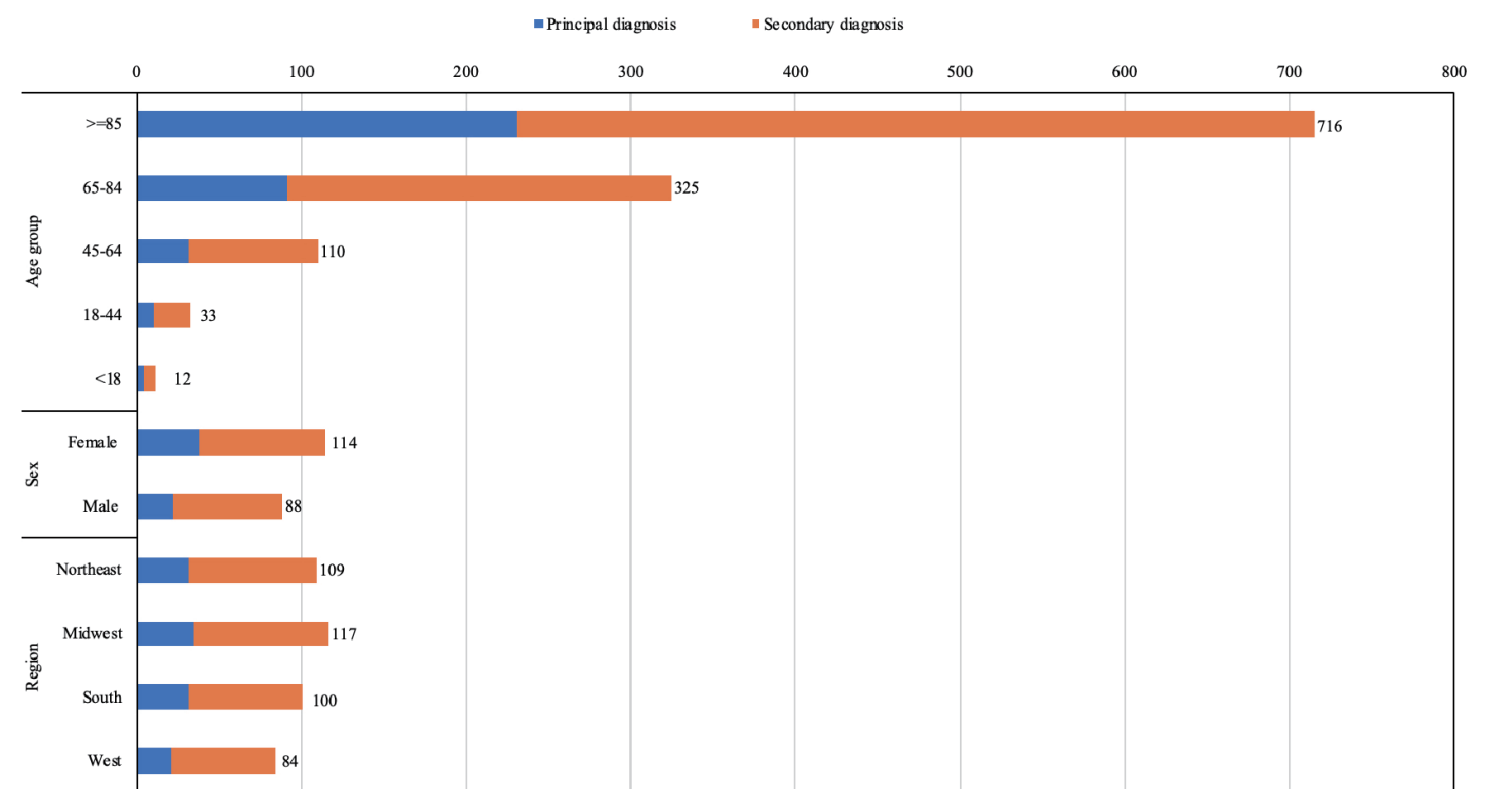

Figure 3. Rate of CDI hospitalizations per 100,000 population, by age group, sex, and region, 2017. CDI: Clostridium difficile infection.

100,000 hospitalizations).

\section{Rates of CDI hospitalizations per 10,000 all-cause hospi- talizations}

The CDI hospitalization rates per 10,000 all-cause hospital discharges by age, sex, and region are depicted in Figure 4.
Patients 85 years and older had the highest rate of CDI hospitalizations (159 per 10,000 hospitalizations). Like population-based rates, there was a progressive increase in the CDI hospitalization rates with each successive age group. There was no significant difference in the hospitalization rate by sex. Midwest had highest rate of CDI hospitalizations (99 per 10,000 hospitalizations) and South had the lowest rate ( 88 per 10,000 hospitalizations). However, the differences between

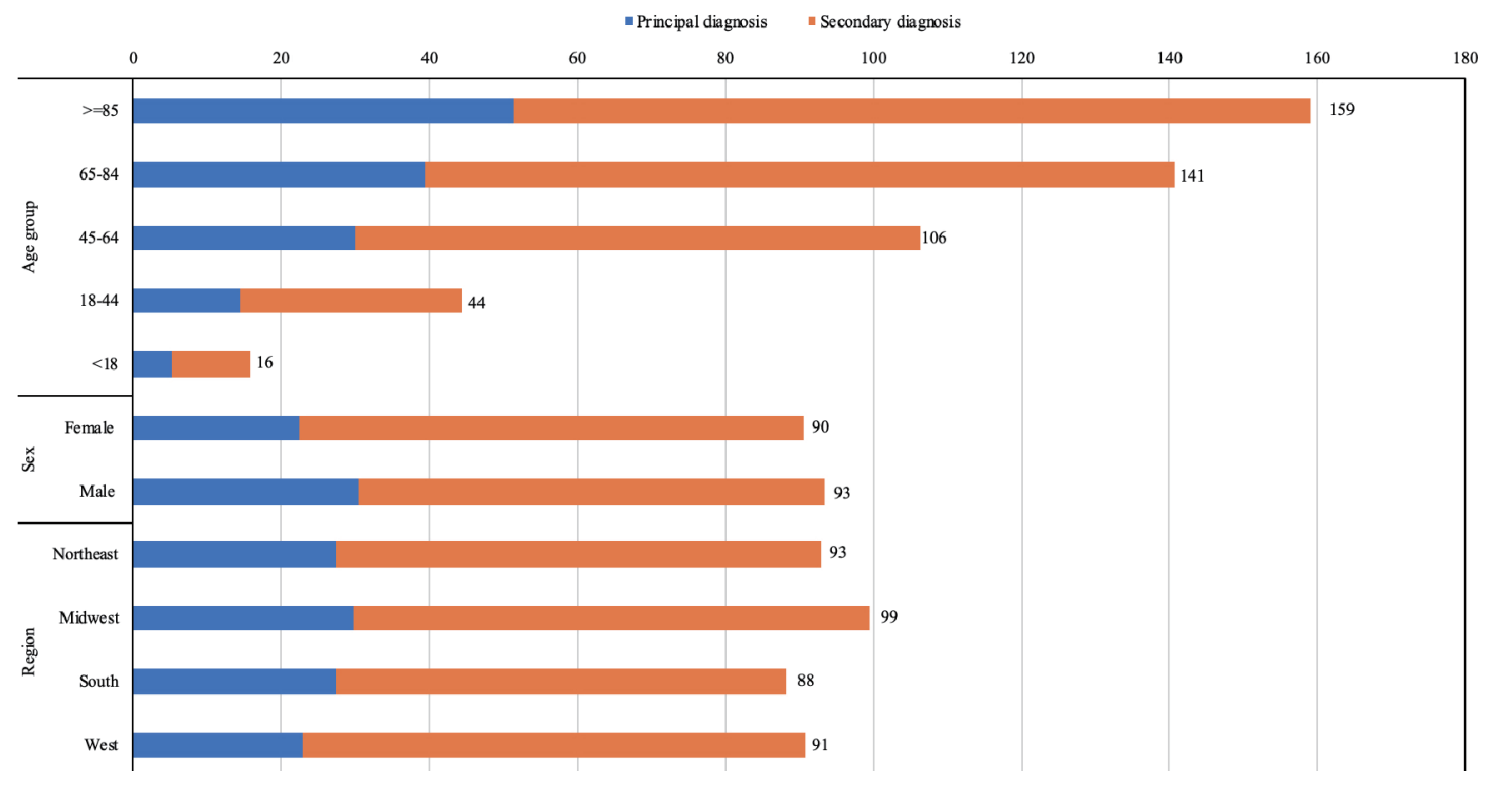

Figure 4. Rates of CDI hospitalizations per 10,000 all-cause hospital stays, by age group, sex, and region, 2017. CDI: Clostridium difficile infection. 
Table 1. Baseline Characteristics of CDI Hospitalizations in 2017

\begin{tabular}{|c|c|c|c|c|}
\hline & All CDI & Principal CDI & Secondary CDI & $\begin{array}{l}\text { All other hos- } \\
\text { pitalizations }\end{array}$ \\
\hline Number of hospitalizations & $\begin{array}{l}329,460(0.9 \% \\
\text { of all stays })\end{array}$ & $\begin{array}{l}96,585(29.3 \% \text { of } \\
\text { all CDI stays })\end{array}$ & $\begin{array}{l}232,875(70.7 \% \\
\text { of all CDI stays })\end{array}$ & $35,470,000$ \\
\hline Mean age (years) & 64.7 & 64.4 & 64.8 & 49.4 \\
\hline Female patients $(\%)$ & 57.1 & 63.7 & 54.4 & 56.4 \\
\hline \multicolumn{5}{|l|}{ Insurance status (\%) } \\
\hline Medicaid & 13.3 & 12.1 & 13.8 & 24.0 \\
\hline Private insurance & 19.0 & 20.5 & 18.3 & 30.3 \\
\hline Uninsured & 2.1 & 2.4 & 2.0 & 4.1 \\
\hline \multicolumn{5}{|l|}{ Severity of illness } \\
\hline $\begin{array}{l}\text { Percentage with APR-DG risk of mortality } \\
\text { score: major or extreme likelihood of dying }(\%)\end{array}$ & 60.0 & 37.7 & 69.2 & 24.9 \\
\hline Mortality rate (\%) & 6.1 & 1.3 & 8.1 & 1.9 \\
\hline Mean length of stay (days) & 9.9 & 5.4 & 11.8 & 4.6 \\
\hline
\end{tabular}

The rates of hospitalizations with $\mathrm{CDI}$ as the principal diagnosis, secondary diagnosis, and all (principal plus secondary) diagnosis are compared with hospitalizations rates for hospitalizations due to all other causes. CDI: Clostridium difficile infection.

the regional hospitalization rates were smaller in magnitude in comparison to the population-based rates.

\section{Severity of illness}

As shown in Table 1, the death rate was higher in CDI hospitalizations than all other hospitalizations (6.1\% vs. $1.9 \%)$. CDI patients were sicker than all hospitalizations in general. CDI patients had a higher severity illness score (3.1 vs. 2.1), and were more than two times likely to have major or extreme loss of function $(79.8 \%$ vs. $33.2 \%)$ compared to all other hospitalizations. Also, CDI patients were more than two times at major or extreme likelihood of dying (60.0\% vs. $24.9 \%)$. Patients with a secondary diagnosis of CDI had a higher risk of major or extreme loss of function $(91.7 \%$ vs. $51.0 \%)$, and were at a higher risk of major or extreme likelihood of dying $(69.2 \%$ vs. $37.7 \%$ ).

\section{Rates of comorbidities associated with CDI}

The common comorbidities associated with CDI are depicted in Table 2. Fluid and electrolyte disturbance was the most common comorbidity associated with CDI hospitalizations $(63.3 \%)$. Sixty-four point nine percent of hospitalizations with CDI as a principal diagnosis and $62.6 \%$ of hospitalizations with CDI as a secondary diagnosis had fluid and electrolyte disturbance. Renal failure was the second most common co- morbidity associated with CDI hospitalizations (33.4\%). Septicemia $(30.7 \%)$ and septic shock $(9.9 \%)$ were the third and fourth most common comorbidities, respectively. Septicemia was around 24 times more common in hospitalizations with CDI as a secondary diagnosis than those with CDI as a principal diagnosis (42.6\% vs. 1.8\%). Septic shock was 23 times more common in hospitalizations with CDI as a secondary diagnosis than those with CDI as a principal diagnosis $(13.8 \%$ vs. $0.6 \%)$. The other common comorbidities were ileus $(3.5 \%)$, ascites $(3.5 \%)$, hypoalbuminemia $(2.4 \%)$, and peritonitis $(2.0 \%)$. Toxic megacolon and bowel perforation were seen in less than $1 \%$ of CDI hospitalizations.

\section{Discussion}

The rate of CDI hospitalizations per 100,000 US population significantly dropped from 2010 to 2017. The rate of CDI hospitalizations increased with increasing age. CDI patients were more than two times at major or extreme likelihood of dying in comparison to patients admitted for all other diagnoses. Fluid imbalance, electrolyte disturbances, and renal failure are the most common comorbidities associated with CDI hospitalizations.

\section{CDI hospitalizations characteristics}

CDI-related hospitalizations in 2017 accounted for $0.9 \%$ of 


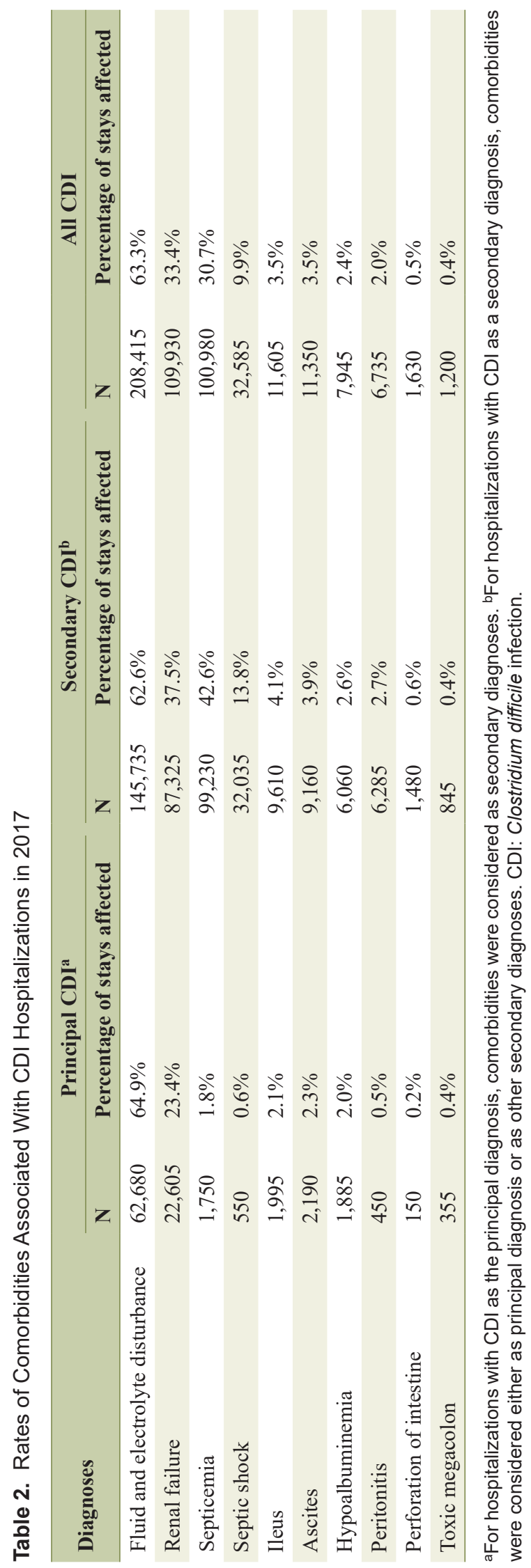

all hospitalizations in 2017, which is similar to an estimate from 2009 from the HCUP [22]. The HCUP data estimate from 2009 found 336,600 hospitalizations that were CDI-related, and 329,460 CDI-related hospitalizations were reported in our findings [22]. From 2010 to 2017, our study found a $1.7 \%$ increase in all CDI-related hospitalizations (Fig. 1). Of the CDI-related hospitalizations, our study reports that $29.3 \%$ had CDI as a principal diagnosis and $70.7 \%$ of the hospitalizations had CDI as a secondary diagnosis. In 2009, HCUP data showed that $33 \%$ of CDI-related hospitalizations had CDI as a principal diagnosis [22]. This means that there was an overall decrease in CDI as a principal diagnosis in CDI-related hospitalizations from 2009 to 2017 . This decline may be attributable to prevention efforts through improvements in infection prevention and antibiotic stewardship, as prevention of CDI has been reported to be a long-standing national priority [23].

The number of all CDI-related hospitalizations increased from 2010 to 2017 by $1.7 \%$ (Fig. 1). However, the rate of all CDI hospitalizations per 100,000 population declined by $3.3 \%$ over this period. One study examining trends for CDI through nucleic acid amplification tests (NAATs) based trends analyses found a decrease in the estimated burden of CDI from 2012 to 2017 , with an overall decrease of $24 \%$ from 2011 to 2017 [23]. This decline may be secondary to antibiotic stewardship efforts, which have been targeted at inpatient settings [23].

\section{Age and sex}

The average age for patients admitted with CDI as a principal or secondary diagnosis was 64.7 years in our study, and patients 85 years and older had the highest rate of CDI hospitalizations, which declined as age decreased. This finding is consistent with results reported by Pechal et al, which showed highest incidence among the elderly followed by adult and subsequently pediatric populations [24]. Numerous studies have reported increased risk of CDI with increased age with overall health being a contributor to CDI risk [25]. CDI incidence has previously been found to be highest in adults who were at least 65 years of age in a national discharge study surveying discharge from 2001 to 2010 [24]. This is likely due to increased use of antibiotics in the elderly [24]. It may also be secondary to proton pump inhibitor (PPI) use, a known risk factor for CDI, which have been reported to be used inappropriately in more than half of a predominantly elderly population in one study and to be prescribed without an appropriate indication or for unknown reasons in some of the population [26]. Longterm PPI use occurs in one of nine older adults, and four out of 10 of these have been reported to have no reason for PPI use [27]. Other possible reasons are: age-related impairment of the immune system, higher prevalence of comorbidities, changes in the gut flora, and frequent health care exposure [24, 28]. Elderly individuals have been shown to have higher in-hospital mortality and longer LOS than adults in previous studies from 2001 to 2010, so the higher prevalence of CDI in this population may warrant more aggressive in-hospital management and monitoring [24]. Young adults likely have lower rated because of stronger immune system, lesser comorbidities, and lesser rate of hospitalizations. 
For all CDI hospitalizations in our study, patients were more likely to be females, and the percent gap between females and males widened when CDI hospitalizations were considered as a primary diagnosis. Hospitalization rates were higher in females than males. Female sex has been reported to be a risk factor for CDI, specifically diarrhea, and CDI incidence has been reported to be higher in women than men [24, 29]. A previous study found that a community-acquired CDI cohort was comprised of more women than men, and the researchers considered that females may be more likely to pursue medical attention, be exposed to antibiotics in an outpatient setting, and thus may develop CDI more than men [30].

\section{Mortality}

In our study, the mortality rate was higher in CDI hospitalizations $(6.1 \%)$ than the mortality rate due to all other hospitalizations (1.9\%), and CDI patients were more than two times at major or extreme likelihood of dying. A literature review from 2005 to 2011 found in-hospital mortality ranging from $8 \%$ to $37.2 \%$ [31]. Higher mortality rates have been reported in the elderly with CDI, and this may reflect the higher risk of CDI development in elderly individuals [24]. Previous studies have reported that severity of infection may be associated with longer LOS, and longer LOS has been found to be associated with increased age [24]. The increased mortality risk may also be secondary to differences in strains, as a prior study showed an increased risk of severe infection and death due to the ribotype 027 strain in older adults compared to younger individuals [32].

\section{Comorbidities}

CDI is a known cause of diarrhea. The hallmark of symptomatic illness is three or more watery, non-bloody stools in a 24-h period [7]. Diarrhea can lead to hypovolemia, electrolyte imbalances, and renal failure secondary to severe dehydration [7]. Thus, fluid and electrolyte disturbances and renal failure were the most common comorbidities associated with CDI hospitalizations. Previous research has found renal failure to be a predictor of complicated CDI when measured on day of diagnosis [33]. Other reports of CDI and renal involvement include the development of immunoglobulin A (IgA) nephropathy after hospital-acquired CDI and development of CDI with a previous diagnosis of acute and chronic renal failure [34]. Additionally, researchers have reported that toxin B from $C$. difficile increases water permeability in collecting duct cells by translocating a water channel, and that $C$. difficile may initiate cell death resulting in acute renal failure [34].

\section{Strengths and limitations}

The population used for our study is drawn from the largest publicly available all-payer inpatient healthcare database. Our study also examines several years of data and numerous de- mographics of CDI admissions, offering a comprehensive and thorough contemporary overview of CDI hospitalizations in the USA. We found that the rate of CDI hospitalizations decreased by $3.3 \%$ from 2010 to 2017 , women were more likely to be affected than men, and patients 85 years of age and above had the highest rate of hospitalizations. When CDI is a secondary diagnosis, major loss of function, extreme likelihood of dying, septicemia, and septic shock were more common in comparison to CDI as a principal diagnosis. The presence of CDI as a comorbid condition at the time of hospitalization for other principal diagnoses or development of CDI during a hospitalization for other principal diagnoses significantly increases the risk of in-hospital morbidity and mortality.

However, there are certain limitations that should be noted. Data from the NIS is subject to biases associated with retrospective studies. Moreover, the NIS reports information on hospitalizations rather than individual patients; thus, patients admitted multiple times would be included more than once in the data set. The NIS also does not include information about the severity of diagnosis at the time of admission, and it uses ICD-10 codes to report hospitalizations, which may have coding errors.

\section{Conclusions}

CDI is a major healthcare burden in the USA, and has been reported to be on the rise despite continued efforts aimed at combating CDI. The aim of this study was to provide contemporary rates of hospitalizations, characteristics of hospitalizations, and rates of comorbid conditions for CDI hospitalizations using data from the NIS. Our study found that almost $1 \%$ of all hospitalizations were for CDI in 2017. The average age for patients admitted with CDI as a principal or secondary diagnosis was 64.7 years, which was almost 20 years older when compared with hospitalizations due to other causes. Patients 85 years and older had the highest rate of CDI hospitalizations, and patients less than 18 years of age had the lowest rate of hospitalizations. A progressive increase in the CDI hospitalization rates was found with increasing age. The hospitalization rates were higher in females than males. Fluid and electrolyte disturbance and renal failure were the two most common comorbidities associated with CDI hospitalizations. Presence of concurrent diseases during CDI hospitalizations results in increased morbidity and mortality. We found that older adults and women are at a higher risk of developing CDI than their younger and male counterparts. Hence, prospective studies should be designed to investigate and understand the reasons behind higher CDI prevalence in these patient groups and develop effective strategies to reduce rates of CDI in these groups.

\section{Supplementary Material}

Suppl 1. Population estimate files and description of variables. Suppl 2. ICD-10-CM codes and CCS codes used for data analysis. 


\section{Acknowledgments}

None to declare.

\section{Financial Disclosure}

None to declare.

\section{Conflict of Interest}

None to declare.

\section{Informed Consent}

Not applicable.

\section{Author Contributions}

Conception and design: Dhanshree Solanki, Shantanu Solanki and Asim Kichloo. Administrative support: Dhanshree Solanki. Provision of data: Asim Kichloo. Collection and assembly of data: Asim Kichloo. Data analysis and interpretation: Shantanu Solanki and Asim Kichloo. Manuscript writing and final approval of manuscript: all authors.

\section{Data Availability}

The authors declare that data supporting the findings of this study are available within the article.

\section{References}

1. Chalmers JD, Akram AR, Singanayagam A, Wilcox MH, Hill AT. Risk factors for Clostridium difficile infection in hospitalized patients with community-acquired pneumonia. J Infect. 2016;73(1):45-53.

2. Slimings C, Riley TV. Antibiotics and hospital-acquired Clostridium difficile infection: update of systematic review and meta-analysis. J Antimicrob Chemother. 2014;69(4):881-891.

3. Lessa FC, Gould CV, McDonald LC. Current status of Clostridium difficile infection epidemiology. Clin Infect Dis. 2012;55(Suppl 2):S65-70.

4. Guh AY, Kutty PK. Clostridioides difficile Infection. Ann Intern Med. 2018;169(7):ITC49-ITC64.

5. Abdullatif VN, Noymer A. Clostridium difficile infection: an emerging cause of death in the twenty-first century. Biodemography Soc Biol. 2016;62(2):198-207.

6. Lessa FC, Mu Y, Bamberg WM, Beldavs ZG, Dumyati GK, Dunn JR, Farley MM, et al. Burden of Clostridium difficile infection in the United States. N Engl J Med.
2015;372(9):825-834

7. Ofosu A. Clostridium difficile infection: a review of current and emerging therapies. Ann Gastroenterol. 2016;29(2):147-154.

8. Johnson S. Combating hospital-acquired C. diff. Nurs Manage. 2018;49(3):16-22.

9. Zilberberg MD, Shorr AF, Kollef MH. Increase in adult Clostridium difficile-related hospitalizations and casefatality rate, United States, 2000-2005. Emerg Infect Dis. 2008;14(6):929-931.

10. Redelings MD, Sorvillo F, Mascola L. Increase in Clostridium difficile-related mortality rates, United States, 19992004. Emerg Infect Dis. 2007;13(9):1417-1419.

11. Overview of National (Nationwide) Inpatient Sample (NIS). Accessed: November 7, 2020. https://www.hcupus.ahrq.gov/nisoverview.jsp.

12. NIS Database Documentation. Accessed: November 7, 2020. https://www.hcup-us.ahrq.gov/db/nation/nis/nisdbdocumentation.jsp.

13. Healthcare Cost and Utilization Project Data Use Agreement Course. Accessed: March 25, 2020. https://www. hcup-us.ahrq.gov/DUA/dua_508/DUA508version.jsp.

14. Population and Housing Unit Estimates. 2020. Accessed: February 7, 2021: https://www.census.gov/programs-surveys/popest.html.

15. NIS Description of Data Elements. 2008. Accessed: February 7, 2021. https://www.hcup-us.ahrq.gov/db/vars/ i10_dxn/nisnote.jsp.

16. International Statistical Classification of Diseases and Related Health Problems (ICD). Accessed: February 7, 2021. https://www.who.int/standards/classifications/classification-of-diseases.

17. Clinical Classifications Software Refined (CCSR). 2020. Accessed: February 7, 2021. https://www.hcup-us.ahrq. gov/toolssoftware/ccsr/ccs_refined.jsp.

18. Khadka S, Solanki D, Singh J, Kichloo A, Patel NR, Sharma P, Chugh S, et al. Trends and outcomes of venous thromboembolism in adult hospitalizations with acute myeloid leukemia: analysis of nationwide inpatient sample from 2010 to 2014. Postgrad Med. 2021;133(2):160165.

19. Kichloo A, Solanki S, Haq KF, et al. Association of nonalcoholic fatty liver disease with gallstone disease in the United States hospitalized patient population. World Journal of Gastrointestinal Pathophysiology. 2021;12:1424.

20. Solanki S, Chakinala RC, Haq KF, Singh J, Khan MA, Solanki D, Vyas MJ, et al. Paralytic ileus in the United States: A cross-sectional study from the national inpatient sample. SAGE Open Med. 2020;8:2050312120962636.

21. Introduction to the HCUP National Inpatient Sample (NIS). Accessed: November 20, 2020. https:/www.hcupus.ahrq.gov/db/nation/nis/NIS_Introduction_2016.jsp, and https://www.hcup-us.ahrq.gov/db/nation/nis/NIS_Introduction_2017.jsp.

22. Clostridium difficile Infections (CDI) in Hospital Stays, 2009. 2012. Accessed: February 7, 2021. https://www. hcup-us.ahrq.gov/reports/statbriefs/sb124.jsp.

23. Guh AY, Mu Y, Winston LG, Johnston H, Olson D, Farley 
MM, Wilson LE, et al. Trends in U.S. Burden of Clostridioides difficile Infection and Outcomes. N Engl J Med. 2020;382(14):1320-1330.

24. Pechal A, Lin K, Allen S, Reveles K. National age group trends in Clostridium difficile infection incidence and health outcomes in United States Community Hospitals. BMC Infect Dis. 2016;16(1):682.

25. Olsen MA, Stwalley D, Demont C, Dubberke ER. Increasing age has limited impact on risk of Clostridium difficile infection in an elderly population. Open Forum Infect Dis. 2018;5(7):ofy160.

26. Choudhry MN, Soran H, Ziglam HM. Overuse and inappropriate prescribing of proton pump inhibitors in patients with Clostridium difficile-associated disease. QJM. 2008;101(6):445-448.

27. Wallerstedt SM, Fastbom J, Linke J, Vitols S. Long-term use of proton pump inhibitors and prevalence of diseaseand drug-related reasons for gastroprotection-a cross-sectional population-based study. Pharmacoepidemiol Drug Saf. 2017;26(1):9-16.

28. Asempa TE, Nicolau DP. Clostridium difficile infection in the elderly: an update on management. Clin Interv Aging. 2017;12:1799-1809.

29. Fekety R, McFarland LV, Surawicz CM, Greenberg RN, Elmer GW, Mulligan ME. Recurrent Clostridium difficile diarrhea: characteristics of and risk factors for patients enrolled in a prospective, randomized, double-blinded trial. Clin Infect Dis. 1997;24(3):324-333.

30. Khanna S, Pardi DS, Aronson SL, Kammer PP, Orenstein R, St Sauver JL, Harmsen WS, et al. The epidemiology of community-acquired Clostridium difficile infection: a population-based study. Am J Gastroenterol. 2012;107(1):89-95.

31. Mitchell BG, Gardner A. Mortality and Clostridium difficile infection: a review. Antimicrobial Resistance and Infection Control. 2012;1:20.

32. Miller M, Gravel D, Mulvey M, Taylor G, Boyd D, Simor A, Gardam M, et al. Health care-associated Clostridium difficile infection in Canada: patient age and infecting strain type are highly predictive of severe outcome and mortality. Clin Infect Dis. 2010;50(2):194-201.

33. Bauer MP, Hensgens MP, Miller MA, Gerding DN, Wilcox MH, Dale AP, Fawley WN, et al. Renal failure and leukocytosis are predictors of a complicated course of Clostridium difficile infection if measured on day of diagnosis. Clin Infect Dis. 2012;55(Suppl 2):S149-153.

34. Arrich J, Sodeck GH, Sengolge G, Konnaris C, Mullner M, Laggner AN, Domanovits H. Clostridium difficile causing acute renal failure: case presentation and review. World J Gastroenterol. 2005;11(8):1245-1247. 